\title{
Structural studies of complicated natural products by new NMR techniques*
}

\author{
Haruo Seto \\ Institute of Molecular and Cellular Biosciences, University of Tokyo, Tokyo 113-0032, \\ Japan
}

Abstract: Three NMR techniques which are prepared by modification of the conventional HMBC, named decoupled HMBC (D-HMBC), 3D-HMBC and constant time HMBC (CT$\mathrm{HMBC}$ ), are proposed. These techniques are superior to $\mathrm{HMBC}$ in respect to signal to noise ratio and resolution, and enable one to observe very small long range ${ }^{13} \mathrm{C}-{ }^{1} \mathrm{H}$ couplings.

Heteronuclear multiple bond correlation (HMBC)[1] is one of the most powerful NMR techniques for structural studies of complicated natural products. Since $\mathrm{HMBC}$ detects ${ }^{13} \mathrm{C}-{ }^{1} \mathrm{H}$ long range correlations by the most sensitive NMR nucleus $\left({ }^{1} \mathrm{H}\right)$ by using an inverse detection, its sensitivity is quite high and enables the natural product chemists to deal with samples available in tiny quantities for structural studies. This technique, however, suffers from low sensitivity or does not give satisfactory results in some cases due to several reasons. In order to overcome these problems, we have developed new NMR techniques prepared by modification of the conventional HMBC. In this article, decoupled HMBC (D-HMBC) [2], 3D-HMBC [3] and constant time HMBC (CT-HMBC) [4] will be explained.

\section{DECOUPLED HMBC (D-HMBC)}

When ${ }^{1} \mathrm{H}$ signals appear as broad lines due to complicated splitting, HMBC suffers from a considerable decrease of the sensitivity. As a consequence, the detection of cross peaks becomes difficult with, for example, complicatedly split methylene proton signals. This problem arises when the power mode data processing causes canceling of anti-phase signal components. The situation is particularly worse when the separation of these components is small and when their signal shapes are broad. Decoupled HMBC (D-HMBC) [2] which is useful to overcome such problems gives a spectrum with improved signal-tonoise ratio.

The pulse sequences of D-HMBC and HMBC are compared in Fig. 1.

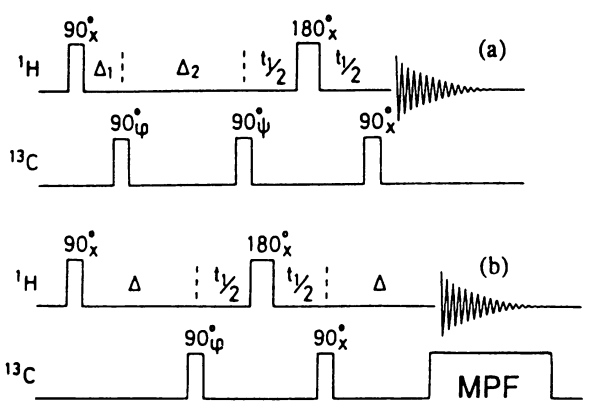

Fig. 1 Pulse sequences of (a) HMBC and (b) D-HMBC.

\footnotetext{
* Invited Lecture presented at the 21st IUPAC International Symposium on The Chemistry of Natural Products (ISCNP-21), Beijing, China, 11-16 October 1998, pp. 1024-1166.
} 
Different from $\mathrm{HMBC}$, an additional delay time $(\Delta)$ is introduced after ${ }^{13} \mathrm{C} 90^{\circ}{ }_{\mathrm{x}}$ pulse to refocus the ${ }^{1} \mathrm{H}$ magnetization, and wide band decoupling (MPF) for the ${ }^{13} \mathrm{C}$ nucleus is employed in D-HMBC. As a result of this modification, a fan-out of the ${ }^{1} \mathrm{H}$ magnetization is prevented, giving in-phase signals with better signal to noise ratio. Thus, the spectra can be acquired in the phase sensitive mode by suppressing the decrease of signal-to-noise ratio due to poor digital resolution.

This pulse sequence is essentially the same as that used for HMQC [5]. It should be noted, however, that since the quaternary carbonyl signals appearing at the lowest field must also be decoupled in this experiment, the decoupling band width required is wider (more than 230 p.p.m.) than that used for HMQC. The low pass J-filter $\left(90^{\circ} \varphi\right.$ pulse for ${ }^{13} \mathrm{C}$ nucleus in the $\mathrm{HMBC}$ pulse sequence) aiming to suppress the cross peaks due to the direct $\mathrm{J}_{\mathrm{C}-\mathrm{H}}$ correlation is not utilized in this case, but it can be implemented if necessary.

Figure 2 compares the HMBC and D-HMBC spectra of an antibiotic monazomycin [6]. It is seen that several cross peaks appear only in the D-HMBC spectrum with suppression of $t_{1}$ noise. In addition, it should be noted that although the cross peak between the carbonyl carbon $(\mathrm{C}-1)$ and oxyrnethine proton (H-47) is weak in Fig. 2b (HMBC), its intensity is increased by approximately twofold in Fig. 2a (DHMBC). The spectra also reveal that the better resolution is obtainable with D-HMBC.

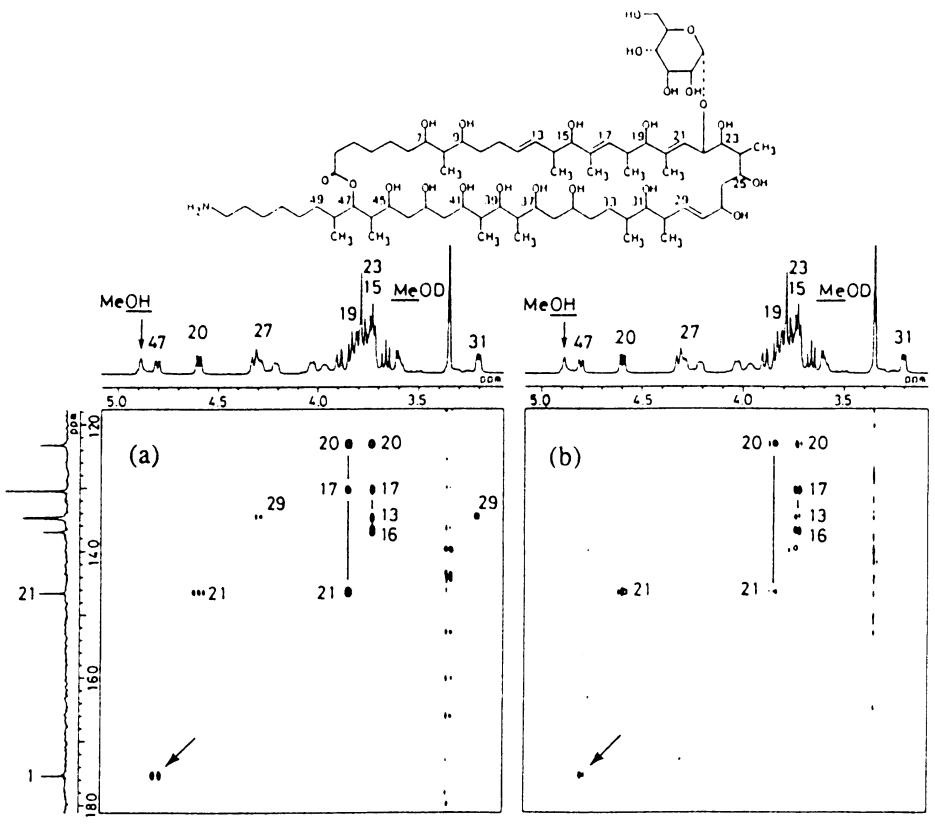

Fig. 2 D-HMBC (a) and HMBC (b) spectra of monazomycin.

D-HMBC also facilitates to observe ${ }^{13} \mathrm{C}-{ }^{1} \mathrm{H}$ long range couplings with small coupling constants. For example, when applied to structural analysis of a thiopeptide compound promothiocin $\mathrm{B}$ [7], long range couplings between ${ }^{13} \mathrm{C}$ and ${ }^{1} \mathrm{H}$ separated by four or five bonds were observed when the delay time was set to $120 \mathrm{~ms}$ or $500 \mathrm{~ms}$ as shown in Fig. 3 (dotted arrows and solid arrows, respectively). In addition, very small long range couplings through three bonds (smaller than $2 \mathrm{~Hz}$ ) which could not be observed by HMBC were detected by D-HMBC. The success in observing such small long range coupling correlations enabled to connect partial structures established by conventional NMR techniques, as shown in Fig. 3 .

\section{D-HMBC}

In order to obtain good HMBC spectra, one of the experimental parameters, delay time $(\Delta)$ must be set to a proper value by considering several parameters such as the magnitude of long-range ${ }^{1} \mathrm{H}-{ }^{13} \mathrm{C}$ coupling constants (for ${ }^{3} \mathrm{~J}_{\mathrm{C}-\mathrm{H}}$ ) and splitting patterns of protons used for detecting ${ }^{1} \mathrm{H}-{ }^{13} \mathrm{C}$ cross peaks. These parameters, however, are depending on the relationships between a given proton and its long-range coupled carbons, and therefore, it is impossible to select the 'almighty' delay time value which will give 


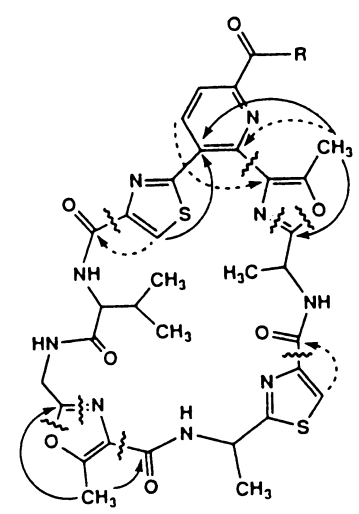

Fig. 3 Long-range couplings observed in the D-HMBC spectra of promothiocin B.

satisfactory results to all ${ }^{1} \mathrm{H}_{-}{ }^{13} \mathrm{C}$ long-range relations. As a compromise, the delta value is generally set to $\Delta=60 \mathrm{~ms}$ for ordinary HMBC experiments resulting in the decrease of the sensitivity for detecting some ${ }^{1} \mathrm{H}-{ }^{13} \mathrm{C}$ long-range relations. The low sensitivity of $\mathrm{HMBC}$ is also raised by multiple couplings of a given proton with several adjacent protons, this being especially striking with methylene proton signals in a complicated proton spin system.

In order to overcome these problems, a new NMR technique, 3D-HMBC, was developed [3]. Its pulse sequence is shown in Fig. 4.

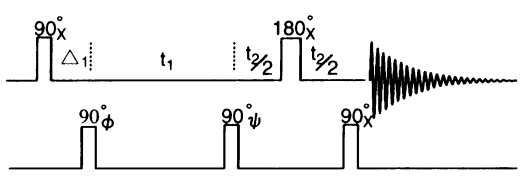

Fig. 4 Pulse sequence of 3D-HMBC.

The great improvement for signal detection is exemplified in Fig. 5, which compares the 2D- and 3DHMBC spectra of monazomycin. In the 2D-HMBC spectrum (Fig. 5, right), only weak cross peaks are
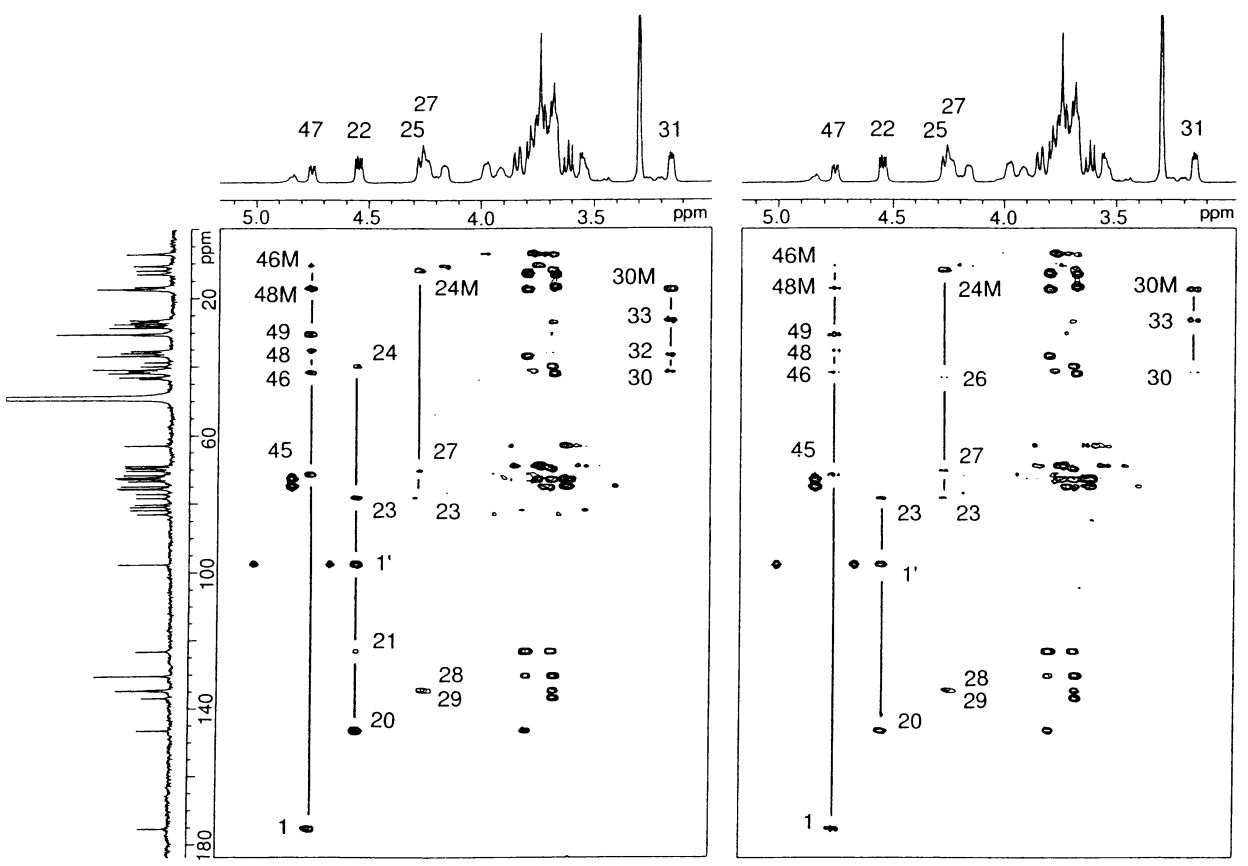

Fig. 52D- and 3D-HMBC spectra of monazomycin. 
observed between a double doublet proton signal, H-22 and C-1', C-20 and C-23. The reason for failure to observe other cross peaks was apparently due to the setting of the delay time $(\Delta)$. In contrast, the 3DHMBC spectra which does not require the setting of such delay time $(\Delta)$ and covers the delay time region from $20 \mathrm{~ms}$ to $80 \mathrm{~ms}$, shows strong cross peaks, for example, from $\mathrm{H}-22$ to C-20, C-23, C-24 and C-1' signals. These results clearly show that the $3 \mathrm{D}-\mathrm{HMBC}$ technique is a usefw method when the information on the connectivity around broad protons signals is not obtainable due to complex splitting patterns of the concerned signals and due to fast transverse relaxation of methylene signals. It should be noted that both spectra were taken in the same experimental time.

\section{CONSTANT TIME HMBC (CT-HMBC)}

One problem of $\mathrm{HMBC}$ is that $\mathrm{J}$-modulation due to ${ }^{1} \mathrm{H}-{ }^{1} \mathrm{H}$ coupling during the $t_{1}$ period causes line broadening of the ${ }^{13} \mathrm{C}$-signals in the $\mathrm{F}_{1}$ dimension. This undesirable effect sometimes results in the difficulty for analyzing ${ }^{13} \mathrm{C}-{ }^{1} \mathrm{H}$ cross peaks of complicated molecules with poorly separated carbon signals.

In order to overcome this problem, we have developed a new technique, constant time HMBC (CTHMBC) [4]. This method gives results better than or at least equal to the conventional HMBC technique in respect to signal resolution and sensitivity, and therefore its use in place of the HMBC technique is highly recommended in all cases for structural studies of complicated molecules.

Figure 6 shows the pulse sequences of new techniques CT-HMBC- 1 and CT-HMBC-2. CT-HMBC-1 is analogous to $\mathrm{HMBC}$ except for the introduction of constant time evolution period [8,9] $\left(\left(\Delta_{3}-\mathrm{t}_{1} / 2\right)+\mathrm{t}_{1} /\right.$ 2) before and after $180^{\circ}$ x pulse. The purpose of incorporating the constant time is to keep constant the $\mathrm{J}$ modulation effect due to homonuclear ${ }^{1} \mathrm{H}-{ }^{1} \mathrm{H}$ coupling during $2 \mathrm{D}$ evolution time $\left(\mathrm{t}_{1}\right)$ by inverting the sign of magnetization during the precession time $\left(\mathrm{t}_{1} / 2+\left(\Delta_{3}-\mathrm{t}_{1} / 2\right)\right)$ after $180^{\circ}{ }_{\mathrm{x}}$ pulse to refocus only the chemical shifts. The net precession time for the full duration (constant time evolution period) is independent of $\mathrm{t}_{1}$. The effect of ${ }^{1} \mathrm{H}-{ }^{1} \mathrm{H} \mathrm{J}$-modulation thus remains unchanged during the constant time period and splitting of the cross peaks by ${ }^{1} \mathrm{H}^{-1} \mathrm{H}$ coupling in the $\mathrm{F}_{1}$ dimension is suppressed.

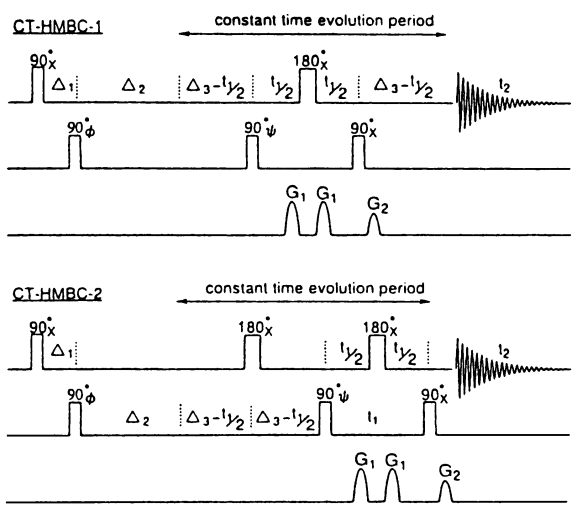

Fig. 6 Pulse sequences of CT-HMBC-1and CT-HMBC-2.

However, HMBC magnetization is modulated by long range ${ }^{13} \mathrm{C}-{ }^{1} \mathrm{H}$ couplings even during the constant time period before $180^{\circ}$ x pulse in the CT-HMBC- 1 experiment. In the CT-HMBC-2, the following constant time period is introduced to give a new pulse sequence; $\left(\Delta_{3}-t_{1} / 2\right)-180^{\circ}-\left(\Delta_{3}-t_{1} / 2\right)$. As a result of this modification, not only $\mathrm{J}$-modulation due to ${ }^{1} \mathrm{H}-{ }^{1} \mathrm{H}$ coupling but also ${ }^{13} \mathrm{C}-{ }^{1} \mathrm{H}$ couplings are suppressed to give better separation than that achieved by CT-HMBC-1.

In order to show actually the advantage of the CT- HMBC technique over the conventional HMBC, partial HMBC, CT-HMBC-1 and CT-HMBC-2 spectra of portmicin [10] are compared in Fig. 7. The cross peaks for $\mathrm{H}-23 / \mathrm{C}-24 \mathrm{M}$ are tilted in the $\mathrm{HMBC}$ spectrum due to ${ }^{1} \mathrm{H}-{ }^{1} \mathrm{H}$ coupling. On the other hand, ${ }^{1} \mathrm{H}-{ }^{1} \mathrm{H}$ couplings are suppressed in the CT-HMBC-1 and CT-HMBC-2 experiments to afford the spectra decoupled in the $\mathrm{F}_{1}$ dimension. The cross peaks in the CT-HMBC-1 spectrum show swelling due to long range ${ }^{13} \mathrm{C}-{ }^{1} \mathrm{H}$ coupling as compared to the CT-HMBC-2 spectrum where a triplet pattern is clearly 

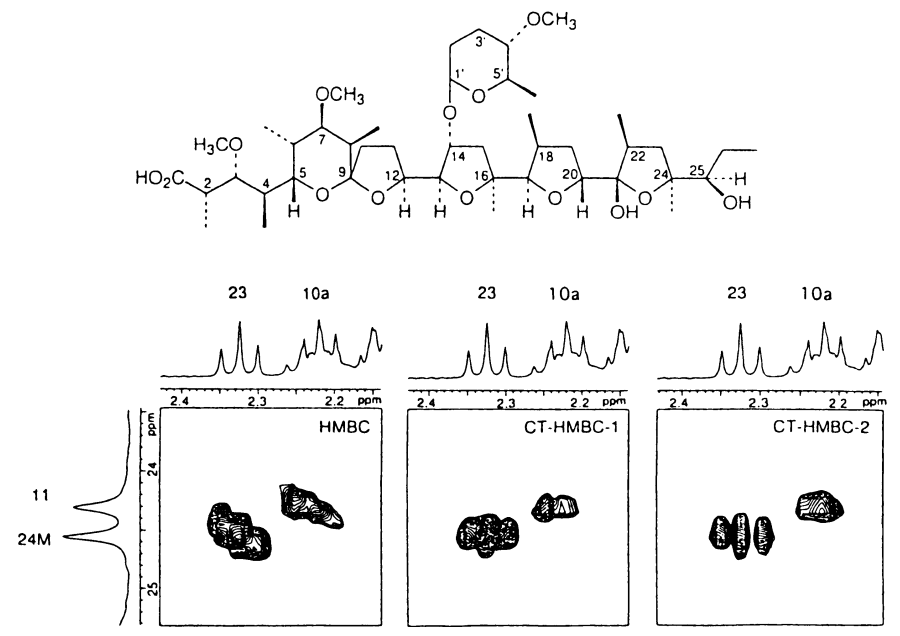

Fig. 7 Partial HMBC, CT-HMBC-1 and CT-HMBC-2 spectra of portmicin.

observed. These results show the apparent advantage of the CT-HMBC technique over the conventional HMBC method.

\section{REFERENCES}

1 M. F. Summers, L. G. Marzilli, A. Bax. J. Am. Chem. Soc. 108, 4285 (1986).

2 K. Furihata, H. Seto. Tetrahedron Lett. 36, 2817 (1995).

3 K. Furihata, H. Seto. Tetrahedron Lett. 37, 8901 (1996).

4 K. Furihata, H. Seto. Tetrahedron Lett. 39, 7337 (1998).

5 A. Bax, S. Sabramanian. J. Magn. Reson. 67, 565 (1986).

6 H. Nakayama, K. Furihata, H. Seto, N. Otake. Tetrahedron Lett. 22, 5217 (1981).

7 B.-S. Yun, T. Hidaka, K. Furihata, H. Seto. J. Antibiot. 47, 510 (1994).

8 A. Bax, R. Freeman. J. Magn. Reson. 44, 542 (1981).

9 M. Ranee, G. Wagner, O. W. Sørensen, K. Wuthrich, R. R. Ernst. J. Magn. Reson. 59, 250 (1984).

10 H. Seto, K. Furihata, K. Saeki, N. Otake, Y. Kusakabe, C. Xu, J. Clardy. Tetrahedron Lett. 28, 3357 (1987). 